\title{
Reduction of Environmental Impact of Drum Machine Washing
}

\section{Pieter Broer van der Weg (Peter)}

Retired, VU Amsterdam, Shell R \& D Rijswijk, Stork DI Boxmeer, Unilever R \& D Vlaardingen, Vlaardingen, The Netherlands

Email: peter@vdweg.com

How to cite this paper: van der Weg $(\mathrm{Pe}$ ter), P.B. (2020) Reduction of Environmental Impact of Drum Machine Washing. Journal of Applied Mathematics and Physics, 8, 132-157.

https://doi.org/10.4236/jamp.2020.81011

Received: December 7, 2019

Accepted: January 5, 2020

Published: January 8, 2020

Copyright (c) 2020 by author(s) and Scientific Research Publishing Inc. This work is licensed under the Creative Commons Attribution International License (CC BY 4.0).

http://creativecommons.org/licenses/by/4.0/

\section{(c) (i) Open Access}

\begin{abstract}
An improvement of efficiency of the horizontally rotating drum washing machine is possible by using a more open type of drum, essentially without suds in the annulus, by using a pump to wet the clothes during rotation and fall. Modelling and simulation are used to quantify these claims and further optimize the design of the horizontal washing machine. The flow of suds inside the deforming clothes at impact with the drum is calculated. The wash performance is shown to be largely proportional to the open perforation area in the drum. The traditional design uses $1 / 8$ of drum area for the perforation holes. A significant reduction of water, detergent, electrical energy, and wash time, with parity in wash performance, provides a step towards a cleaner and more sustainable future.
\end{abstract}

\section{Keywords}

Drum, Wash, Machine, Design, Efficiency, Poroelastics, Darcy, Simulation, Modelling

\section{Introduction}

Wash performance is a balance between temperature, chemistry, time, and mechanical action [1] [2] [3]. Many of these factors have been researched, quantified, and used to optimize the wash performance to the satisfaction of the consumer.

Solid and oily dirt removal occurs when mechanical drag forces from the wash fluid exceed the interfacial retaining forces that attach the dirt to the fabric. For good and fast wash action, fast flow of liquid relative to the fabric is needed. The largest drag forces and relative drainage flow occur at the impact of the falling fabric within a rotating drum, at beating and slapping, or when the articles are scrubbed with a brush during hand washing. 
Surfactants in the suds facilitate the lowering of the interfacial tension which dislodges dirt, keeps the dirt in suspension, and might even assist in the mechanics of the washing process by creating repeated air patches (foam) within the clothes, although those air patches might also reduce wash efficiency.

In a horizontal rotating drum laundry appliance, the fabric cannot lose water fast enough to create quick and good washing due to the limited space of the holes in the drum and due to the presence of water in the annulus under the holes at the moment of fall impact of the laundry articles within the drum. The term water means the aqueous detergent solution (suds) during washing. But it may also describe cleaning water during a rinsing step.

An improvement would be to use a very open grating of the drum, suck water from the annulus to replace the water around the drum by air and re-inject the water through the open grating and/or inside the drum to rewet the articles. This increased washing machine efficiency enables significant reduction in use of water, chemicals, time, and energy, at comparable or even better wash performance compared to the traditional appliance.

Tighter control by politicians and governments, demands a sustainable future with minimal negative environmental impact. We foresee shortages in supplies, energy, and freshwater. Our improved wash machine design, with significantly reduced water, chemicals and energy consumption may contribute to that economic and sustainable future.

Much of this research has been developed and reported internally by the author in 2004-2006 at HPC Unilever.

\section{The Horizontal Rotating Drum Washing Machine}

\subsection{The Classical Design}

The present commercial design of the rotating drums uses many (900 - 4000) holes of $2-4 \mathrm{~mm}$ diameter in the drum such that the wash liquid may pass (drain and imbibe) between the inner space of the drum and the annulus space around the drum. The total area of the open holes in the drum of typical commercial washing machines is less than $1 / 8$ of the total area of the outer cylindrical drum. This creates a significant barrier for flow: the liquid flow out of the articles falling on the inside bottom area of a horizontally rotating drum is retarded and reduced as the water has to flow sideways before it can pass the holes in the drum. The flow of water through the holes is further retarded due to the presence of water in the annulus under the holes. If an article hits the cylindrical inner metal surface of the drum, not directly over a hole, the water is ejected sideways at lower speed in multiple directions over the inside of the drum surface. Furthermore, in the present design, the presence of suds inside the drum further muffles the fall impact of the articles.

\subsection{The Improved Design}

If the article hits an open maze (say a $1-2 \mathrm{~mm}$ mesh gauze), with air instead of 
water on the outside, the water can continue to flow in the direction of the fall, almost perpendicularly to the surface of the drum, creating a faster flow, and hence, better and faster cleaning compared to an article that would hit a solid surface of the drum. The mesh of the drum gauze must be small enough to avoid trapping attachments on clothes (e.g. buttons, cords, strings.)

In centrifuges, the drum is mounted often vertically rotating, in washing machines the drum may rotate horizontally or vertically. In the horizontal drum wash process, the rpm is so low (typically $25-60 \mathrm{rpm}$ ) that the wet articles fall due to gravity and impact with the lower part of the drum to enhance the liberation of wash liquid from the inside of the fabrics of the articles. The trajectory of a small article, represented as a point mass, is calculated in Appendix A. The optimum rpm for a given drum diameter, representing the largest change in momentum of a point mass on impact, is calculated in Appendix B. See Figure 1 for a typical example of a $48 \mathrm{~cm}$ diameter horizontal drum at optimum $40.8 \mathrm{rpm}$.

The direction of the rotation of the drum is reversed several times during the wash. This creates a pattern that is the mirror image of the pattern given in Figure 1 . Such a reversal rearranges the clothes and unwinds any knots of clothes that may have formed during the rotation in one direction, getting a more even wash effect on the clothes.

In spinning and centrifuging, the rpm are so high that a large part of the liquid is expelled from the fabric through the holes by the centrifugal force on the

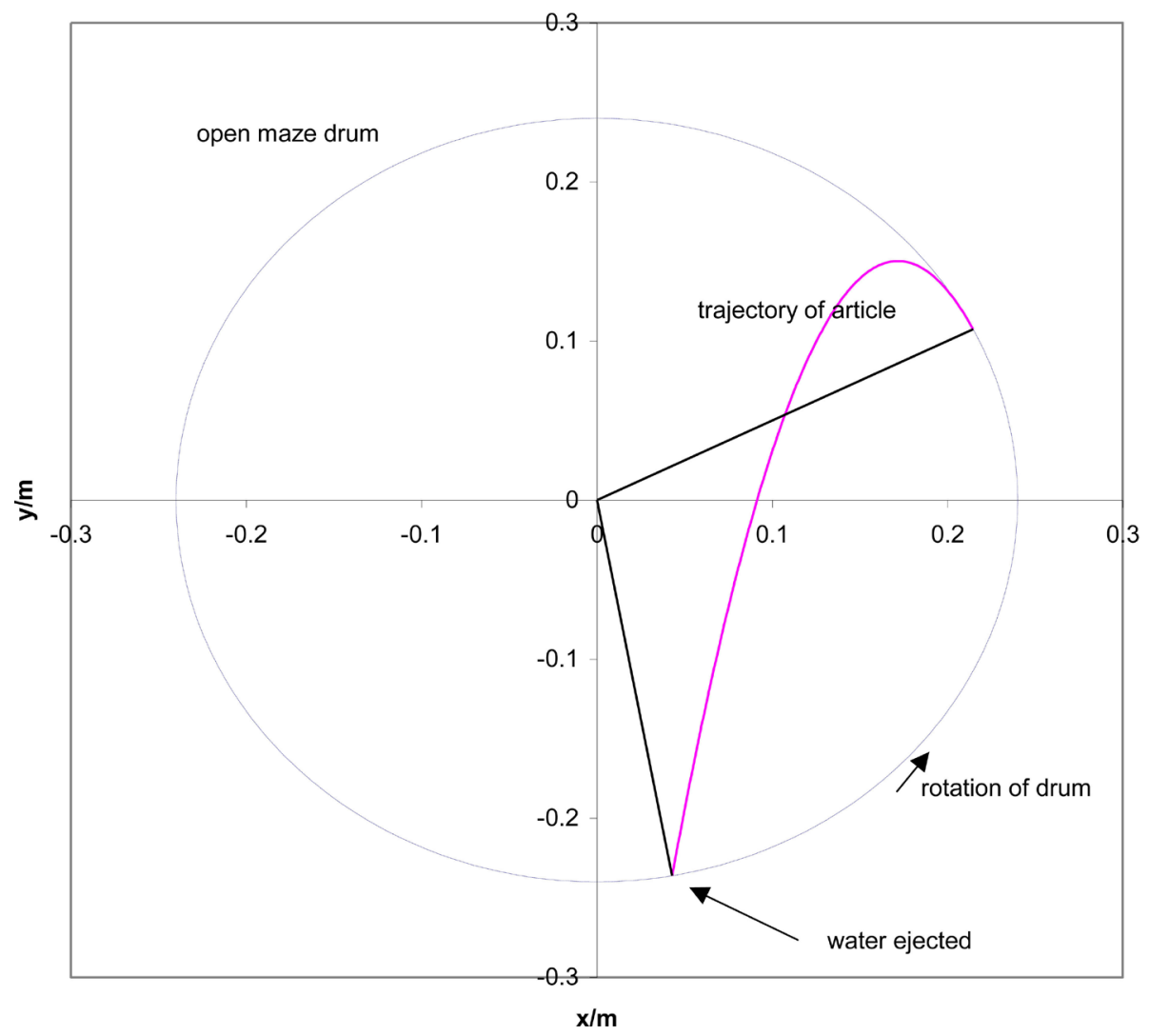

Figure 1. Side view of the drum perpendicular to axis of rotation. 
water in the articles pressed to the inside of the cylindrical circumference of the drum.

We propose a much more open design of the drum (preferably more than $15 \%$ open space, preferably as open as possible, limited at the high side by strength considerations), such that the liquid may flow more freely in and out in the wash and spin processes. This will speed up the wash and spin process while reaching superior cleaning and drying of the clothes during the wash and spin-drying/centrifuging process respectively. The size of the mesh of the drum should be small enough that buttons and small articles do not get trapped in the holes.

To make the process more favourable in a horizontal axis washing machine, we propose to fill the drum holes and the annulus with air instead of water, by using less water and pumping the water more quickly away from the annulus. That water would muffle the impact and thus, reduce the release of water when clothes impact the drum after the fall in a horizontal drum. Moreover, the drag on the drum is reduced, as the drum no longer rotates through the stagnant water in the annulus between the drum and the housing.

Less water means that for the same dose, the concentration of surfactant is increased, creating better wash-process efficiency, and more efficient batch rinsing. Alternatively, the dose may be reduced, still maintaining the same or even a higher chemical concentration, while fewer chemicals are consumed. Very high concentrations of surfactants and bleach lead to damage of dyes in clothes. For surfactants, a concentration of the order of the critical micellar concentration during the wash is optimum.

During the wash process, the water is re-injected, to re-wet the clothes before they fall again. The circulation speed (refresh rate) of the water is improved compared to conventional design, improving the speed of mixing of chemicals, faster heating, and improving the efficiency of the wash process. The water is preferably injected through the side surface of the drum, just beyond the point of fall contact, such that the clothes have sufficient time and can take up the water again on their way up to the next fall. By choosing the injection more or less in line with the direction of rotation, the beam helps to rotate the drum. The open design of the drum surface also allows the re-injection of the water with sufficient force (as a beam or spray) through the open drum surface to add to the cleaning efficiency, to add to the lift force, and to loosen the articles from the drum surface. The extra force of the injected water in the direction of the motion, as well as the absence of drag through water outside the drum, relaxes the design strength of the motor. The re-injected water (suds) acts against the centrifugal force of the rotation and the downward force of gravity. Injection of water through the open surface of the drum will expand the compacted layer and stretch the fabric before the next fall. Hence, articles will wet more efficiently than in the conventional design and the injected water will allow the articles to better relax their shape. The injection might be directed more in an upward di- 
rection, in order to align with the rotation of the drum, helping the rotation and reducing the moment exerted by the clothes normally pressed against the inside of the drum. Some of the suds will hit the open maze, creating a spray. Such a spray may enhance the formation of foam that may be beneficial (consumers like foam) or not beneficial (more antifoam needed). Air pockets-from the foamin the pores of, and in between, the clothes might help to repeat the pressure-induced flow of suds impacting on the drum. This might repeat the wash effect on impact several times within the clothes. Without foam, the flow of suds in the pores of the article only occurs near the impact plane. With foam, this process may repeat several times, in each subsequent fluid pocket. Hence, foam might not only be aesthetically pleasing but might also improve the wash performance.

The exact injection point/line will depend on the rotational direction of the drum. Maybe the 3 or 4 ribs usually seen in conventional designs for drum strengthening and to drag the clothes around can be removed. They reduce the wash efficiency: Clothes in contact with the ribs are rotated more towards the middle of the drum, and are more likely to fall due to reduced centrifugal force, thus releasing their water less efficiently.

As the annulus is mostly filled with air, if needed, small ribs can be mounted on the outside in order to strengthen the drum. Since the cleaning potential is greater for a larger inner drum radius and lower rpm, a maximum radius of the drum is recommended in the design of the drum.

The rougher surface of the mesh of the drum surface will create a more even drag force on the clothes as they fall onto the drum and are then dragged along with the rotation of the drum. The slip at the first moment of contact with the drum is reduced, reducing the surface wear of the articles and creating a deeper wash effect (slip only partially cleans the surface). The more even drag will distribute the wash effect more evenly over the whole contact time, more evenly cleaning the article, and more evenly distributing the washing force (the bending/acceleration of the clothes and the release of water) over the area of the falling article. The article arrives at the drum a factor 2 faster than the rotation speed of the drum; in other words, the article is rumpled up 2 times when dragged along with the drum. The enhanced grip of the open grating will help to get a more even spread of the clothes, without sliding over the surface of the drum. The shorter wash with higher wash efficiency per cycle might effectively lower the damage and wear of the clothes, but that can only be assessed in a prototype.

The design of the open drum structure might require new and stronger materials (like carbon fibre enforced plastics) and new engineering.

Due to the forced reinjection of suds through the open grating of the drum no waiting step is needed between reversing rotation to re-soak the clothes, as is needed in conventional designs, speeding up the wash process.

The amount of water to effectively soak the articles is reduced from $7-10$ li- 
tres to say 3 - 4 litres or less for a $5 \mathrm{~kg}$ wash. The chemical formulation detergent dose can be reduced by the same factor. Less water means proportional reduction of time and energy spent for heating. Also, foam inside the fabric may reduce water use.

The active circulation of the wash liquor will reduce stagnant areas and volumes where chemicals can accumulate without contributing to the wash process.

The wash time can be reduced for the same wash result: a simple bench-top test of dropping standard dirty clothes 200 times (e.g. $11 \mathrm{~cm} \times 11 \mathrm{~cm}$ WFK30d and EMPA104 monitors stitched on a washing glove) from a height of $80 \mathrm{~cm}$ on a plate or a $44 \%$ open grid $(2 \times 2 \mathrm{~mm}$ pores, $1 \mathrm{~mm}$ wire, e.g. $2 \mathrm{~mm}$ mesh), while re-soaking in between (with $5 \mathrm{~g} / \mathrm{L} \mathrm{EC} \mathrm{A} \mathrm{no-phosphate} \mathrm{standard} \mathrm{detergent,} \mathrm{roughly}$ at $40^{\circ} \mathrm{C}$ ) simulating the mechanics of the wash process, proved a reduction of $9 \%$ - $22 \%$ wash time for the more open structure. The wash performance was measured with a Minolta reflectometer in duplicates.

Also, in the hand wash, the use of a washing board with a fine open grating drains more suds on impact and therefore, improves the wash performance per stroke.

Rinsing becomes more efficient, with shortened time and less water when the amount of water per rinsing step is reduced.

\section{Modelling and Simulating Wash Performance in Drum Washing}

The trajectory of a small article in a horizontal rotating drum is derived in Appendices $\mathrm{A}$ and $\mathrm{B}$. The optimum trajectory with maximum wash efficiency has the largest velocity change on impact with the drum. For a $48 \mathrm{~cm}$ diameter drum, that optimum is reached at $41 \mathrm{rpm}$, with a trajectory as sketched in Figure 1 and a speed change on impact of $\Delta v=3.3 \mathrm{~m} / \mathrm{s}$.

\subsection{Single Phase Flow in Porous Media}

The simulation of single-phase flow from gravitational compaction in deformable porous ("poroelastic") media is described in [4]. We will use a similar theory to model the fluid flow draining from clothes on fall impact on the drum. The impact of soaked fabric with the drum creates a surge of water draining from the article. Note: Here the fluid flow is pressure-induced and in the same direction as fabric flow.

The continuous incompressible suds are assumed aqueous and Newtonian. The isotropic dispersed medium is a fibrous cloth compacting during fluid drainage. The fluid wets the cloth. Drainage stems from the simple fall impact of a wetted cloth inside the drum. Temperature is assumed constant. The flow rates are so slow that the fluid follows Stokes flow and Darcy's law applies for the flow through the clothes. The flow near the surface of the drum, in the first few millimetres, is approximated as 1-dimensional and perpendicular to the drum surface. We assume that the cloth reacts elastically on compaction. Foam is as- 
sumed absent in the fabric, but if present, would probably further enhance the wash performance.

The flow of liquid in porous media is described by the (linear) Darcy's law $q=-\frac{k}{\mu} \frac{\partial P}{\partial z}$. Here $k$ is the permeability of the fabric and $\mu$ the viscosity of the liquid. Note: In [4] the volume flow rates of fluid and dispersed porous medium were equal and in opposite directions, which led to a factor 2 reduction in Darcy's law.

\subsection{A Simplified Wash Model}

In the model, we assume for simplicity that the article hits a stationary drum perpendicularly in a one-dimensional motion in a vertical $z$-direction with a speed $\Delta \mathrm{v}$. The article is stopped, while the water continues to flow for a while through the grating of the drum, but slowed down by the drag of the fabric. In a simple model, we will assume that the area in the maze of the drum is open and allows free flow of water. The $\mathrm{z}$-coordinate is the height above the drum surface. This single vertical $\mathrm{z}$-coordinate is a simplification of the $(x, y)$ trajectory near impact with the drum. At impact on the drum, an instantaneous pressure excess $\Delta P=\frac{1}{2} \rho(\Delta v)^{2}$ is created in the fluid at the surface of the fabric, driving the liquid through the drum.

While the liquid drains, the fabric is contracted in the vertical direction and a strain is developed to support the pressure gradient $\frac{\partial P}{\partial z}=\varepsilon \frac{\partial S}{\partial z}=-\varepsilon \frac{\partial \phi}{\partial z}$, where $S$ the strain $\delta z / z, \phi$ the porosity, and $\varepsilon$ the compressibility of the fabric. The flow of liquid is directly changing the (liquid) porosity. The conservation of volume requires $\frac{\partial q}{\partial z}=-\frac{\partial \phi}{\partial t}$. Combining these equations, we find $\frac{\partial q}{\partial z}=-\frac{\partial \phi}{\partial t}=\frac{\partial}{\partial z}\left(-\frac{k}{\mu} \frac{\partial P}{\partial z}\right)=\frac{\partial}{\partial z}\left(\frac{k \varepsilon}{\mu} \frac{\partial \phi}{\partial z}\right)$. Hence, we arrive at a differential equation $\frac{\partial \phi}{\partial t}=\frac{\partial}{\partial z}\left(\frac{k \varepsilon}{\mu} \frac{\partial \phi}{\partial z}\right)$. Both permeability $k$ and elasticity $\varepsilon$ are functions of porosity $\phi$. Simple generic functions in our porosity range are for permeability Carman-Kozeny $k=\frac{c \phi^{3}}{a_{g}^{2}} \approx k_{0}\left(\frac{\phi}{\phi_{0}}\right)^{3}\left(\frac{1-\phi_{0}}{1-\phi}\right)^{2}$ and for elasticity the modified heuristic Van Wyk's equation $\varepsilon=\varepsilon_{0}\left(\frac{1-\phi}{1-\phi_{0}}\right)^{3}\left(\frac{\phi_{0}}{\phi}\right)^{4}$, (see [4] [5] [6] [7]), leading to $\frac{\partial \phi}{\partial t}=\frac{k_{0} \varepsilon_{0}}{\mu} \frac{\partial}{\partial z}\left\{\left(\frac{1-\phi}{1-\phi_{0}}\right)\left(\frac{\phi_{0}}{\phi}\right) \frac{\partial \phi}{\partial z}\right\}$. This is a nonlinear second-order partial differential equation (PDE) that can only be solved by numerical simulation for the proper initial and boundary conditions, but for small excursions in porosity is $\frac{\partial \phi}{\partial t} \approx \frac{k_{0} \varepsilon_{0}}{\mu} \frac{\partial^{2} \phi}{\partial z^{2}}$, which is a purely parabolic PDE. This leads to scaling 
$\frac{z^{2}}{t}=\frac{k_{0} \varepsilon_{0}}{\mu}=D_{s}$. The compaction disturbance travels upwards with the square root of time proportional to the square root of a dispersion coefficient of consolidation $D_{s}=\frac{k_{0} \varepsilon_{0}}{\mu}$, similar to the Terzaghi equation for the gravity-induced compaction of fluid saturated soil [8] [9].

Suppose we assume that the material drains from one side in a one-dimensional way and that the material is so thick, or times are so short, that the drainage disturbance does not reach the back of the fabric, within the time span of observation. Standard mathematics leads to a solution $\phi(z, t)=\phi_{0} \operatorname{erf}\left(\frac{z}{2 \sqrt{D_{s} t}}\right)$. This solution is familiar in diffusion and heat conductance modelling. Erf is the Gauss Error function $\operatorname{erf}(x)=\frac{2}{\sqrt{\pi}} \int_{0}^{x} \mathrm{e}^{-u^{2}} \mathrm{~d} u$. The amount of fluid flowing is $q(z, t)=-\frac{k_{0}}{\mu} \frac{\partial P}{\partial z}=-D_{s} \frac{\partial \phi}{\partial z}$. Liquid flows in the direction of the lowest pressure. To make liquid flow out of the porous medium through $z=0$, the pressure on the outside must be lower than inside. Then $q(z, t)=-D_{s} \frac{\partial \phi}{\partial z}=-D_{s} \frac{\partial}{\partial z}\left(\phi_{0} e r f\left(\frac{z}{2 \sqrt{D_{s} t}}\right)\right)$. Mass balance gives
$\int q(z=0, t) \mathrm{d} t=-\int_{0}^{t} D_{s} \phi_{0}\left[\frac{2 \mathrm{e}^{-\frac{z^{2}}{4 D_{s} t}}}{\sqrt{\pi}} \frac{1}{2 \sqrt{D_{s} t}}\right]_{z=0} \mathrm{~d} t$ or
$\frac{Q(z=0, t)}{A}=\int q(z=0) \mathrm{d} t=-\frac{2}{\sqrt{\pi}} \sqrt{D_{s}} \phi_{0} \sqrt{t}$. The liquid continues to flow with time at a diminishing rate. This is the case because it was assumed that the disturbance has not (yet) reached the other side of the medium. In our case, the production of fluid at $z=0$ leads to a compaction shortening of length $\int q(z=0, t) \mathrm{d} t=\Omega(t)=-\frac{2}{\sqrt{\pi}} \sqrt{D_{s}} \phi_{0} \sqrt{t}$, where $\Omega$ the compaction length. This simple model applies in processes like filtration compaction, machine and hand wash. In this simplified gravity compaction model, the compaction shortening $\Omega$ is proportional to $\sqrt{t}$ during the entire time span that the compaction disturbance has not reached the other side. For a contact time of order $(d / \Delta v)$, where $d$ the thickness and $\Delta v$ the impact arrival speed of the article, we expect per impact an amount of liquid per unit area to be drained of order $\Delta q_{\text {impact }}=\frac{2}{\sqrt{\pi}} \sqrt{D_{s}} \phi_{0} \sqrt{\frac{d}{\Delta v}}$.

For a set parameters that would represent cotton, e.g. $\phi_{0}=0.63$, $k_{0}=4 \times 10^{-12} \mathrm{~m}^{2}$ (4 Darcy), $\varepsilon_{0}=14000 \mathrm{~N} / \mathrm{m}^{2}$, article thickness $z=2 \mathrm{~mm}, \mu=1$ $\mathrm{mPas}$, we arrive at an analytical solution as shown in Figure 2.

The $0.62 \mathrm{~ms}$ time of calculation corresponds to the contact time $\tau$ of a $2 \mathrm{~mm}$ thick article at an impact speed of $3.2 \mathrm{~m} / \mathrm{s}$ in a typical open drum washing machine; 


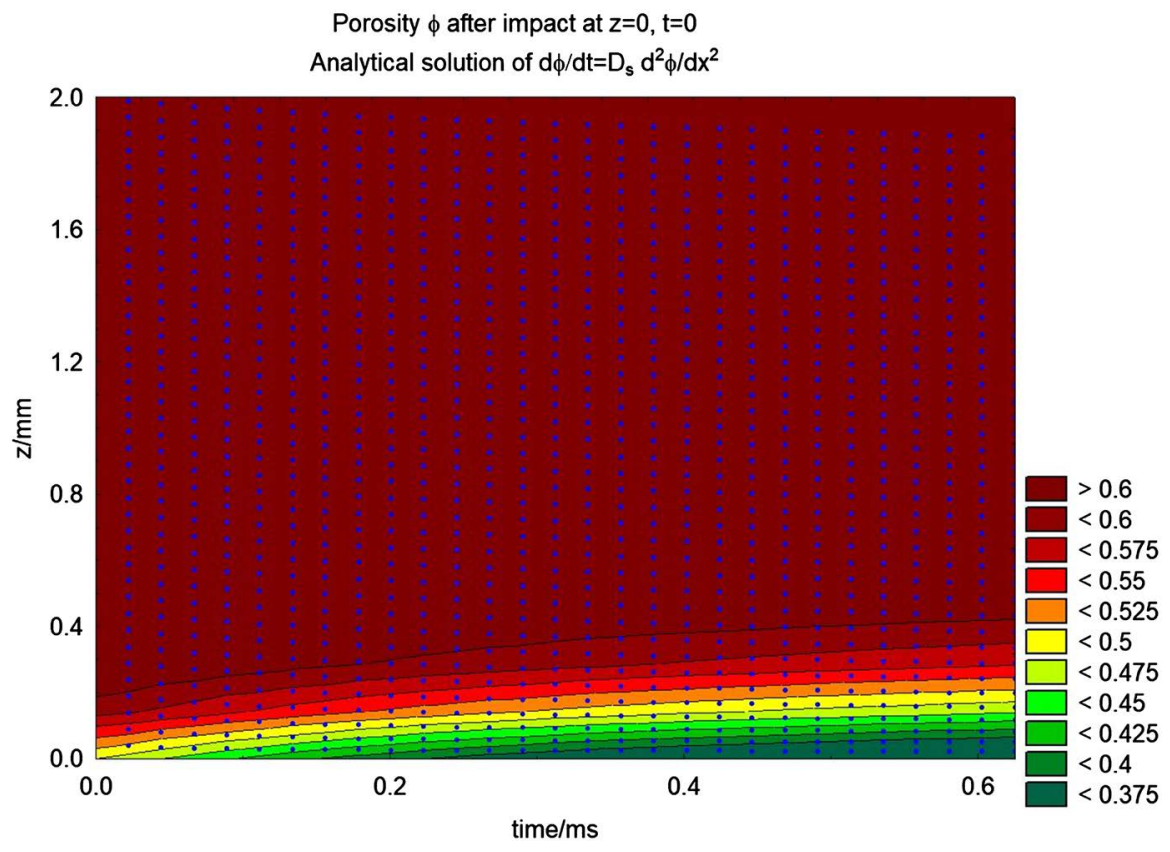

Figure 2. Analytical solution of vertical porosity profile as function of time after impact.

e.g. a time of order $\tau \approx 2 \mathrm{~mm} / 3.2 \mathrm{~m} / \mathrm{s}=0.62 \mathrm{~ms}$. We calculate a contraction from $2 \mathrm{~mm}$ to $1.881 \mathrm{~mm}$, or over $0.12 \mathrm{~mm}$, hence $6 \%$ volume produced per impact. In the 1-d calculation, the height of the article is assumed infinite, but should at least be $2.12 \mathrm{~mm}$. If the article was $2 \mathrm{~mm}$ high, the top level (both fluid and fabric) would have lowered over a distance of $\Omega=0.12 \mathrm{~mm}$ in $0.62 \mathrm{~ms}$. The average Darcy flow rate inside the compaction zone is $0.12 \mathrm{~mm} / 0.62 \mathrm{~ms} \approx 0.19 \mathrm{~m} / \mathrm{s}$. This flow rate is directly responsible for the cleaning action.

\subsection{Simulation of Flow Improvement for an Open Gauze Type of Drum}

We will use a simple explicit simulation with Gauss-Seidel iteration to show the impact of holes compared to impacts on open and closed gratings in a steady state.

We use a 3-dimensional simulation of creeping flow impact to simulate the effectiveness of holes and compare that with the effectiveness of a totally closed and completely open grating impact (which are essentially 1-dimensional simulations).

If we assume that the parameter combination $D_{s}=k \varepsilon / \mu=k_{0} \varepsilon_{0} / \mu$ is a constant, the creeping 3-dimensional simulation of $\phi(z, x, y, t)$ involves the following PDE: $\frac{\partial \phi}{\partial t}=D_{s}\left(\frac{\partial^{2} \phi}{\partial z^{2}}+\frac{\partial^{2} \phi}{\partial x^{2}}+\frac{\partial^{2} \phi}{\partial y^{2}}\right)$. If we now make this equation discrete for steps $\Delta t, \Delta z, \Delta x, \Delta y$ we arrive at

$$
\begin{aligned}
\frac{\phi_{i, j, k, n+1}-\phi_{i, j, k, n}}{\Delta t}= & D_{s}\left(\frac{\phi_{i-1, j, k, n}-2 \phi_{i, j, k, n}+\phi_{i+1, j, k, n}}{\Delta z^{2}}+\frac{\phi_{i, j-1, k, n}-2 \phi_{i, j, k, n}+\phi_{i, j+1, k, n}}{\Delta x^{2}}\right. \\
& \left.+\frac{\phi_{i, j, k-1, n}-2 \phi_{i, j, k, n}+\phi_{i, j, k+1, n}}{\Delta x^{2}}\right) .
\end{aligned}
$$


If we now choose $\Delta x=\Delta z=\Delta y$, then

$$
\begin{aligned}
& \phi_{i, j, k, n+1}-\phi_{i, j, k, n} \\
& =6 D_{s} \frac{\Delta t}{\Delta z^{2}}\left(\frac{\phi_{i-1, j, k, n}+\phi_{i+1, j, k, n}+\phi_{i, j-1, k, n}+\phi_{i, j+1, k, n}+\phi_{i, j, k-1, n}+\phi_{i, j, k+1, n}}{6}-\phi_{i, j k, n}\right) .
\end{aligned}
$$

If we now choose step sizes such that $6 D_{s} \frac{\Delta t}{\Delta z^{2}}=1$, then we arrive at a very simple expression

$\phi_{i, j, k, n+1}=\left(\frac{\phi_{i-1, j, k, n}+\phi_{i+1, j, k, n}+\phi_{i, j-1, k, n}+\phi_{i, j+1, k, n}+\phi_{i, j, k-1, n}+\phi_{i, j, k+1, n}}{6}\right)$. For this step size, the porosity at a node after each time step is simply the average of the surrounding nodes.

Likewise, for 2-dimensional simulation $\phi(z, x, t) ; \Delta t, \Delta z=\Delta x$, and

$4 D_{s} \frac{\Delta t}{\Delta z^{2}}=1$, we find $\phi_{i, j, n+1}=\left(\frac{\phi_{i-1, j, n}+\phi_{i+1, j, n}+\phi_{i, j-1, n}+\phi_{i, j+1, n}}{4}\right)$ and in 1-d for

$2 D_{s} \frac{\Delta t}{\Delta z^{2}}=1$, we find $\phi_{i, n+1}=\left(\frac{\phi_{i-1, n}+\phi_{i+1, n}}{2}\right)$.

When we simulate the behaviour after many time steps, a given porosity distribution at the boundaries, we approach a steady-state solution for porosity distribution in a creeping flow profile. By imposing different boundary conditions, representing the distribution and sizes of the holes in the drum, we may compare the wash efficiency for these distributions. By simulating for the appropriate and fixed number of time steps, we may even compare the flow profiles very close to the time of impact of 0.63 s or at any other time of interest before steady state is reached.

We simulate in 3 -d in a box $(20 \times 20 \times 20)$ with points $(i, j, k, n)$ at $(z=\Delta z * i, \quad x=\Delta x * j, \quad y=\Delta y * k, \quad t=n * \Delta t)$ with appropriate boundary conditions. The article is $2 \times 2 \times 2 \mathrm{~mm}^{3}$ and falls in the $\mathrm{z}$-direction on a grating. We will use the same wetted cotton parameters as before: $k=4 \times 10^{-12} \mathrm{~m}^{2}$, $\varepsilon=14000 \mathrm{~N} / \mathrm{m}^{2}, \mu=10^{-3} \mathrm{~Pa} \cdot \mathrm{s}, \Delta z=\left(2 \times 10^{-3}\right) / 20 \mathrm{~m}$, e.g. $0.1 \mathrm{~mm}$, average wetted $\rho=1.11 \mathrm{~g} / \mathrm{cm}^{3}, 2 \mathrm{~mm}$ thick, and total impact time $t=0.63 \mathrm{~s}$ at impact at $3.21 \mathrm{~m} / \mathrm{s}$, hence, in $3-\mathrm{d}$ is $\Delta t=\frac{\Delta z^{2}}{6 D_{s}}=2.98 \times 10^{-2} \mathrm{~ms}$.

We will show the impact of the size of square holes in the lower plane. We may compare with a virtual completely open surface where water can continue to flow while article is stopped at the surface. In 21 time steps we have simulated up to the impact time $t=0.63 \mathrm{~ms}$. In the 2 -d case $\Delta t=\frac{\Delta z^{2}}{4 D_{s}}=4.46 \times 10^{-2} \mathrm{~ms}$, we need 14 time steps for $t=0.63 \mathrm{~ms}$. In the 1 - $\mathrm{d}$ case $\Delta t=\frac{\Delta z^{2}}{2 D_{s}}=8.93 \times 10^{-2} \mathrm{~ms}$, e.g. 7 time steps for $t=0.63 \mathrm{~ms}$.

The initial porosity is 0.63 . An impact with $3.21 \mathrm{~m} / \mathrm{s}$ will create a pressure of $5724 \mathrm{~N} / \mathrm{m}^{2}$ and locally diminish porosity to 0.2211 when free to drain (into the 
hole).

First, we will simulate 3-d until a steady-state to show the effect of different hole distributions and sizes. By putting a square hole of different sizes in the middle lower boundary plane of the box, we have symmetry in the $x, y$-plane and may represent the flow profile by a vertical cross-section through the middle, for instance for the $\mathrm{x}$-direction.

The first simulation, Figure 3, represents the steady-state for a $2 \mathrm{~mm}^{3}$ wetted cotton box that on a grating with a square hole in the bottom $(z=0)$ for $0<x<$ $2 \mathrm{~mm}$ and $0<y<2 \mathrm{~mm}$, thus an area of $4 \mathrm{~mm}^{2}$, with a porosity of 0.22 (representing the pressure of impact at $3.21 \mathrm{~m} / \mathrm{s}$ ) while the other faces of the box are maintained at a porosity of 0.63 . All surfaces will allow flow in any direction. The behaviour is similar to the steady-state temperature profile in a box of a material of given thermal diffusivity maintained at fixed and different temperatures on the boundaries.

The compression of the wetted cotton means a drain of $18.5 \%$ of pore volume at steady state. The points represent the discrete points used in the simulation.

The second simulation, Figure 4, represents the steady-state for a $2 \mathrm{~mm}^{3}$ wetted cotton box that on a grating with a square hole in the middle of the bottom $(z=0)$ for $0.7<x<1.5 \mathrm{~mm}$ and $0.7<y<1.5 \mathrm{~mm}$, thus an area of $0.64 \mathrm{~mm}^{2}$, with a porosity of 0.22 (representing the pressure of impact at $3.21 \mathrm{~m} / \mathrm{s}$ ) while the other faces of the box are maintained at a porosity of 0.63 .

The compression of the wetted cotton means a drain of $6.23 \%$ of pore volume at steady-state. We indeed observe that the steady-state wash effect is limited to the area of the hole.

The third simulation, Figure 5, represents the profile at a time of $0.65 \mathrm{~ms}$ (21

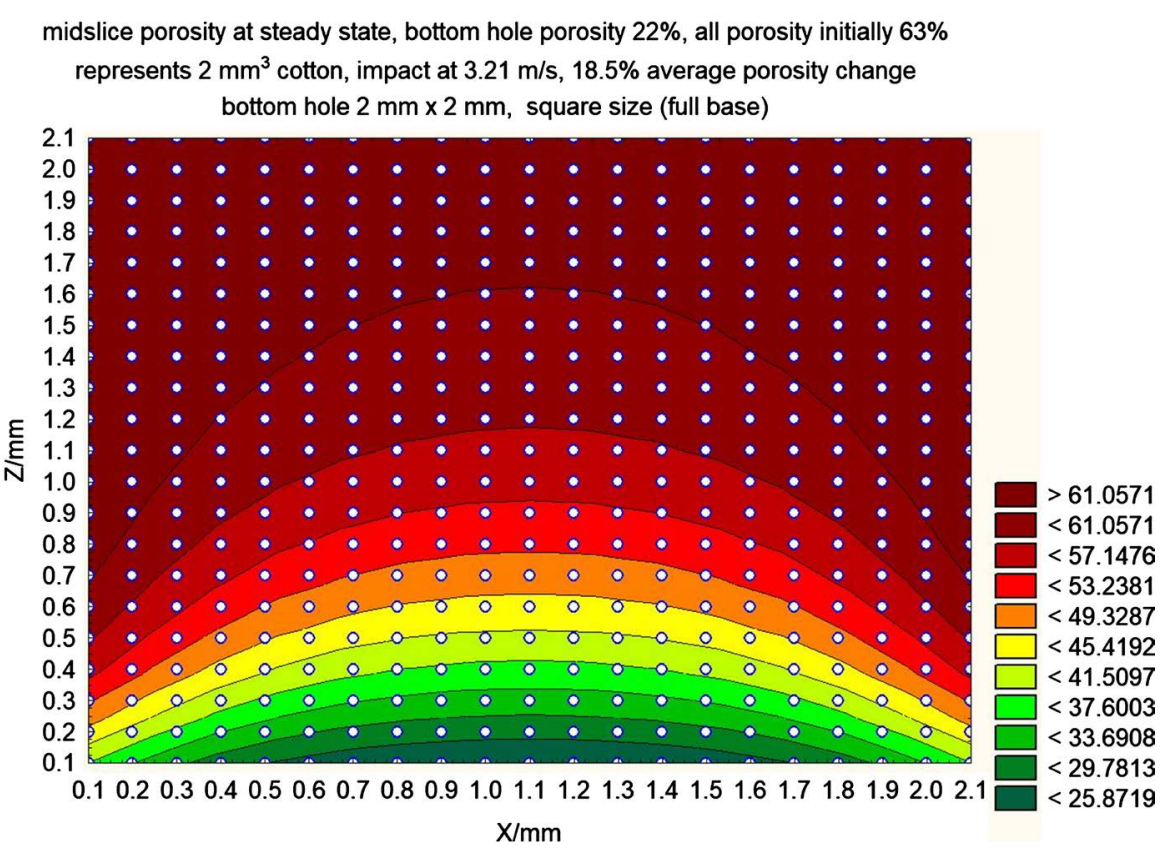

Figure 3. Impact drainage of wetted $2 \times 2 \times 2 \mathrm{~mm}^{3}$ cotton falling at $3.21 \mathrm{~m} / \mathrm{s}$ on an open grating $\left(2 \times 2 \mathrm{~mm}^{2}\right)$ at steady state. 
midslice porosity at steady state, bottom hole porosity $22 \%$, all porosity initially $63 \%$ represents $2 \mathrm{~mm}^{3}$ cotton, impact at $3.21 \mathrm{~m} / \mathrm{s}, 6.23 \%$ average porosity change bottom hole $0.8 \mathrm{~mm} \times 0.8 \mathrm{~mm}$ in middle, square size

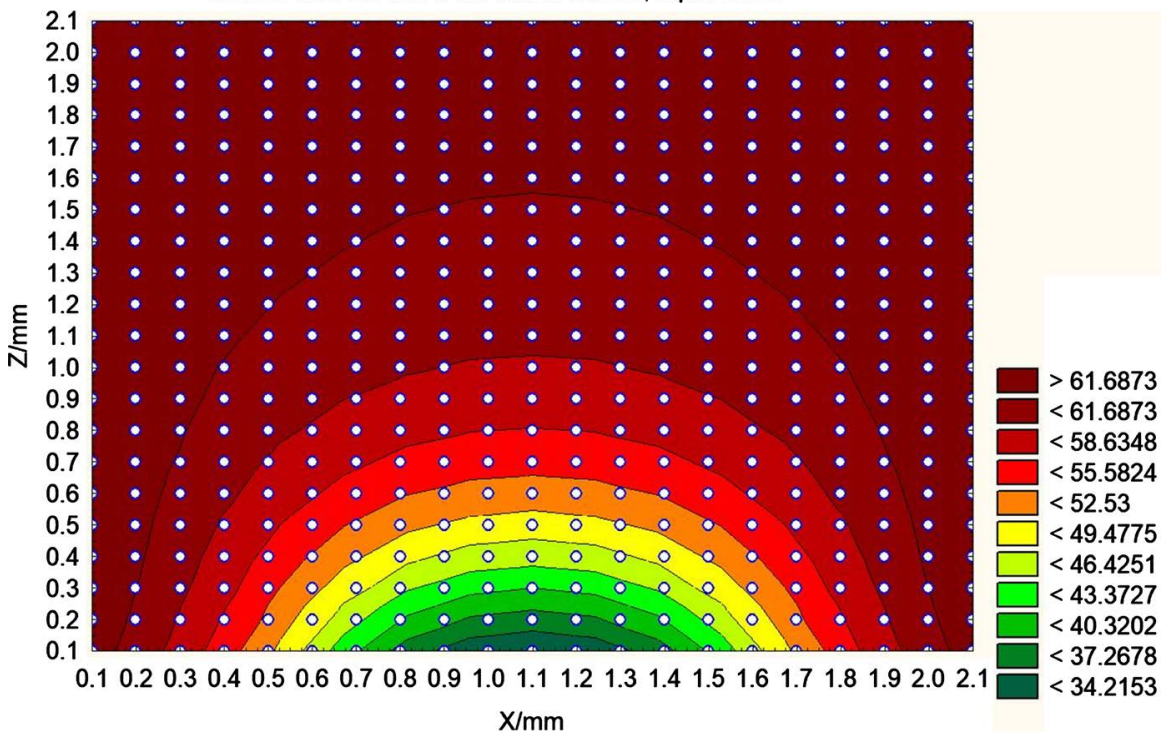

Figure 4. Impact drainage of wetted $2 \times 2 \times 2 \mathrm{~mm}^{3}$ cotton falling at $3.21 \mathrm{~m} / \mathrm{s}$ on a partially closed grating $\left(0.8 \times 0.8 \mathrm{~mm}^{2}\right.$ open $)$ at steady state.

midslice porosity at $0.65 \mathrm{~ms}$, bottom hole porosity $22 \%$, all porosity initially $63 \%$ represents $2 \mathrm{~mm}^{3}$ cotton, impact at $3.21 \mathrm{~m} / \mathrm{s}, 11.77 \%$ average porosity change bottom hole $2 \mathrm{~mm} \times 2 \mathrm{~mm}$, square size (full base)

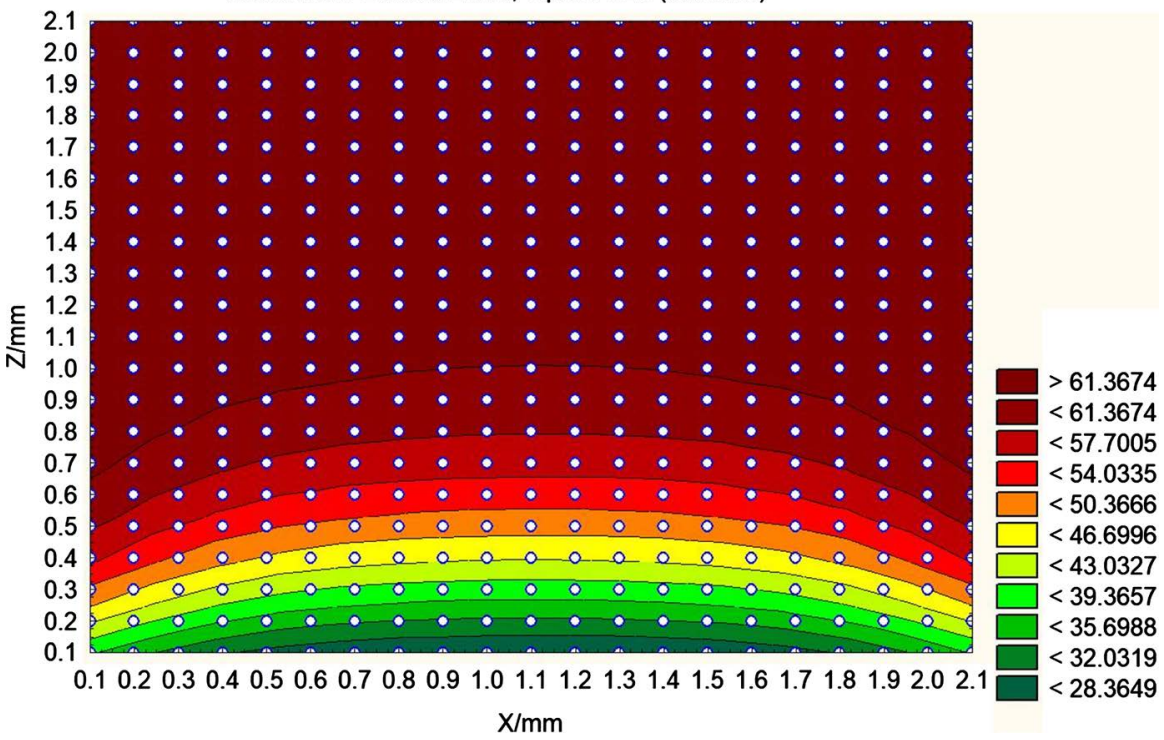

Figure 5. Impact drainage of wetted $2 \times 2 \times 2 \mathrm{~mm}^{3}$ cotton falling at $3.21 \mathrm{~m} / \mathrm{s}$ on a open grating $\left(2 \times 2 \mathrm{~mm}^{2}\right) 0.65 \mathrm{~ms}$ after impact.

time steps) for a $2 \mathrm{~mm}^{3}$ wetted cotton box that on a grating with a square hole in the bottom $(z=0)$ for $0<x<2 \mathrm{~mm}$ and $0<y<2 \mathrm{~mm}$, thus an area of $4 \mathrm{~mm}^{2}$, with a porosity of 0.22 (representing the pressure of impact at $3.21 \mathrm{~m} / \mathrm{s}$ ) while the other faces of the box are maintained at a porosity of 0.63 .

The compression of the wetted cotton means a drain of $11.8 \%$ of pore volume in $0.63 \mathrm{~ms}$. We also observe that most of the wash effect is limited to a penetra- 
tion depth of about half a millimetre.

The fourth simulation, Figure 6, represents the profile at a time of $0.65 \mathrm{~ms}$ (21 time steps) for a $2 \mathrm{~mm}^{3}$ wetted cotton box that on a grating with a square hole in the middle of the bottom $(z=0)$ for $0.7<x<1.5 \mathrm{~mm}$ and $0.7<y<1.5 \mathrm{~mm}$, thus an area of $0.64 \mathrm{~mm}^{2}$, with a porosity of 0.22 (representing the pressure of impact at $3.21 \mathrm{~m} / \mathrm{s}$ ) while the other faces of the box are maintained at a porosity of 0.63 .

The compression of the wetted cotton means a drain of $3.46 \%$ of pore volume in $0.63 \mathrm{~ms}$.

These graphs show that porosity changes, and therefore, efficient washing effects are only observed above and in the near vicinity of the holes with a penetration depth of about half of a millimetre for the $3.21 \mathrm{~m} / \mathrm{s}$ impact of a $2 \mathrm{~mm}^{3}$ box of cotton. Larger areas taken by the holes extend the wash effect over a larger area, although the maximum relative speed in the exit plane of the hole, and therefore the maximum wash effect reached during the wash, remains nearly the same.

The total drainage increases with the fraction of the area taken by the holes of the total area of the impact plane. This indicates that indeed an open area will significantly enhance the speed of the wash: an area increase from $0.8 \times 0.8 \mathrm{~mm}^{2}$ to $2 \times 2 \mathrm{~mm}^{2}$ increases the compression from $6.23 \%$ to $18.5 \%$ (steady-state, e.g. 12 to $13 \mathrm{~ms}$ ) and from $3.46 \%$ to $11.77 \%$ in $0.63 \mathrm{~ms}$ wash time. An open area hole size increase with a factor 6 leads to a factor 3 in the speed of wash; this is significant, but not fully proportional to the area increase. If we add extra holes of the same size in between the existing holes, the wash speed will be nearly proportional to the number of holes.

In these simulations, the side walls did not allow vertical flow (porosity constant

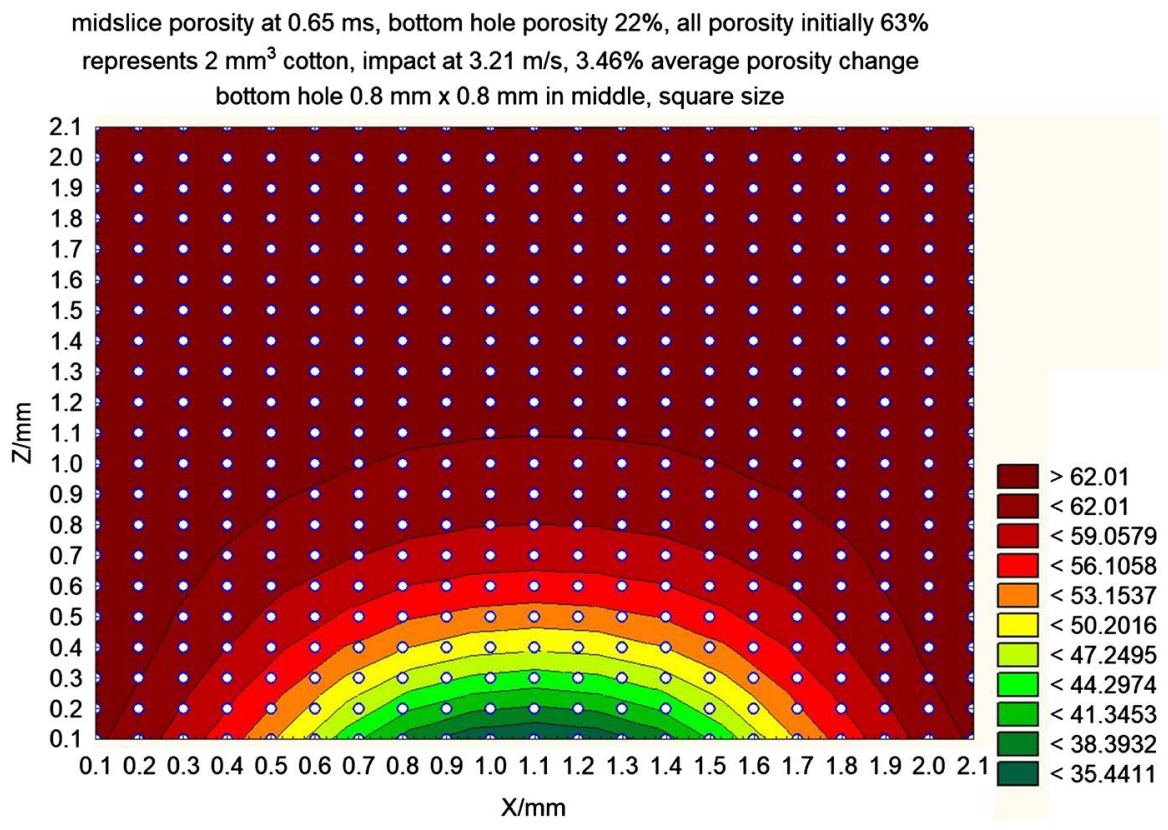

Figure 6. Impact drainage of wetted $2 \times 2 \times 2 \mathrm{~mm}^{3}$ cotton falling at $3.21 \mathrm{~m} / \mathrm{s}$ on a partially closed grating $\left(0.8 \times 0.8 \mathrm{~mm}^{2}\right.$ open $) 0.65 \mathrm{~ms}$ after impact. 
at 63\%). In real washing machines, the side walls allow vertical flow for each unit cell around a hole, and therefore the wash effect for a total open grating is larger. The whole box will then approach the wash efficiency depicted at the centre line of the two "open" grating graphs, further enlarging the difference in wash effect compared to the smaller holes. This is shown in the next simulation, Figure 7, where we have allowed vertical flow in the side planes, but blocked horizontal flow through the side planes, with a full open grating at porosity 0.22 in the bottom plane. This is done by equating, after each step, the porosities in the vertical wall to the porosities in the second cell on the inside, thereby annihilating the horizontal flow through the first cell on the inside, but still allowing the full vertical flow to develop. This results in pure vertical flow everywhere.

We observe compaction to $15.66 \%$, significantly better than the $11.8 \%$ reached in Figure 6 at the same time $(0.65 \mathrm{~ms})$ after impact. The ratio with the $0.8 \times 0.9$ $\mathrm{mm}^{2}$ hole is $3.46 / 15.66=0.22$, approaching the surface ratio of the holes $(0.8 \times$ $0.8) /(2 \times 2)=0.16$. We see that the penetration depth (half of porosity change) is of order $0.4 \mathrm{~mm}$.

These 3-d simulations yield larger compressions than the analytical solution (15.7\% instead of $6 \%)$. This might indicate that the crude 3 -d simulations are not very precise. However, for comparing the relative effect of different hole sizes on the compaction, such simple simulations are very handy. Refining the step size from $20 \times 20 \times 20$ to $30 \times 30 \times 30$ leads to $15.27 \%$ compaction in $0.65 \mathrm{~ms}$, showing that the solution is reasonably stable.

The porosity change means that the fabric is compacted, while liquid is expelled and drained through the bottom face, creating a flow of wash liquor relative to the fabric and thus, creating a washing effect. The flow speed diminishes in the vertical direction and becomes negligible above $0.5 \mathrm{~mm}$ from the bottom depth.

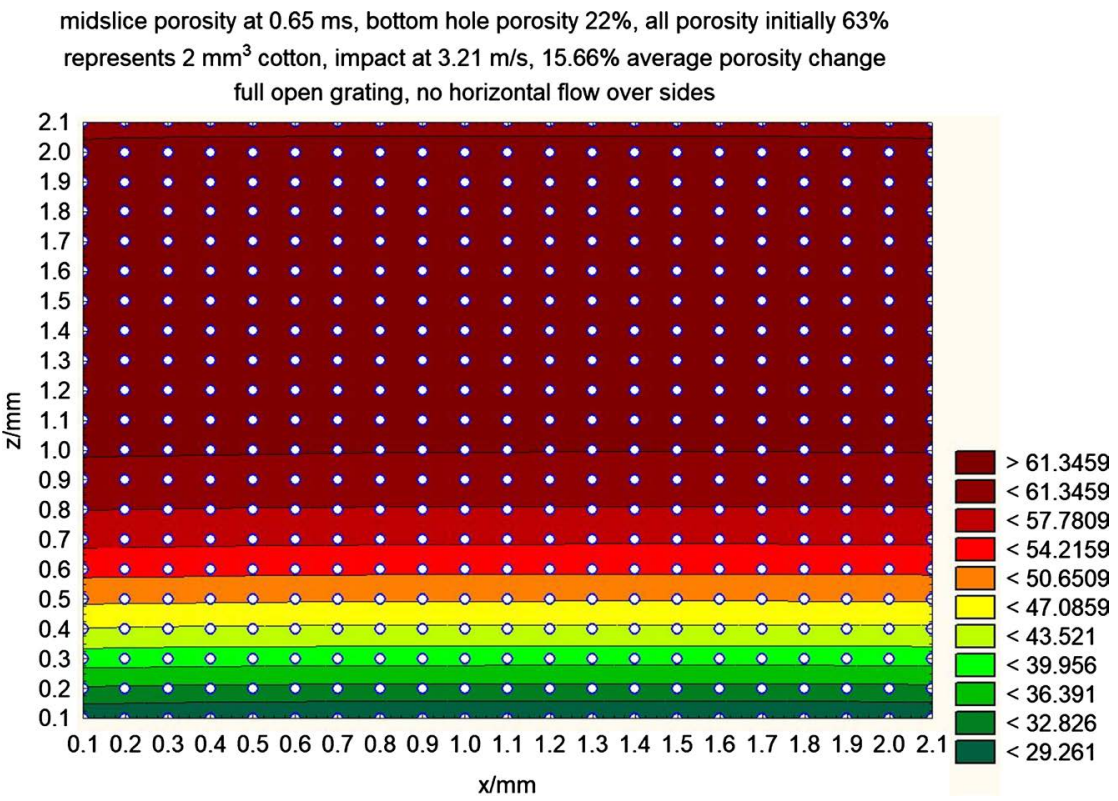

Figure 7. Impact drainage of wetted $2 \times 2 \times 2 \mathrm{~mm}^{3}$ cotton falling at $3.21 \mathrm{~m} / \mathrm{s}$ on an open grating $\left(2 \times 2 \mathrm{~mm}^{2}\right.$ open) $0.65 \mathrm{~ms}$ after impact, with only vertical flow. 
Above that small penetration depth, the fabric and the liquor move at the same speed without any washing effects.

Sample code for the $0.63 \mathrm{~ms}$ simulation of the $0.8 \times 0.8 \mathrm{~mm}^{2}$ hole in a $20 \times 20$ $\times 20$ box of $2 \times 2 \times 2 \mathrm{~mm}^{3}$ box in FORTRAN with no horizontal flow over the vertical boundaries is given in Appendix C. The result is shown in Figure 8.

There is compaction with $3.61 \%$, very similar to the $3.46 \%$ in Figure 7 , where flow over the side boundaries was allowed. We, therefore, conclude that for the holes in the bottom layer, the boundary conditions on the side walls are only of minor influence. Most flow is occurring in the vicinity of the holes, where the disturbance travels inwards from the bottom boundary layer without being affected beyond the circle of influence of say $1 \mathrm{~mm}$.

The conventional designs of the rotating drum use many (900 - 4000) holes of $2-4 \mathrm{~mm}$ diameter in the drum such that the wash liquid may pass (drain and imbibe) between the inner space of the rotary drum and the annulus space around the rotary drum. The total area of the open holes in the drum of typical commercial washing machines is less than $1 / 8(12.5 \%)$ of the total area of the outer cylindrical drum. By designing with an open grating (e.g. a 1 - $2 \mathrm{~mm}$ mesh gauze, easily realizing a 50\% open area) and air below the drum, much faster and even better cleaning is possible.

In real life, the fluid may also drain through the sides of the article, but at a slower speed (by symmetry typically half of the maximum vertical speed), and the fluid must then first on average penetrate a quarter of the thickness of the article. Such wash effects are expected to be of lower efficiency but are essential for the traditional drum with only a small number of small holes.

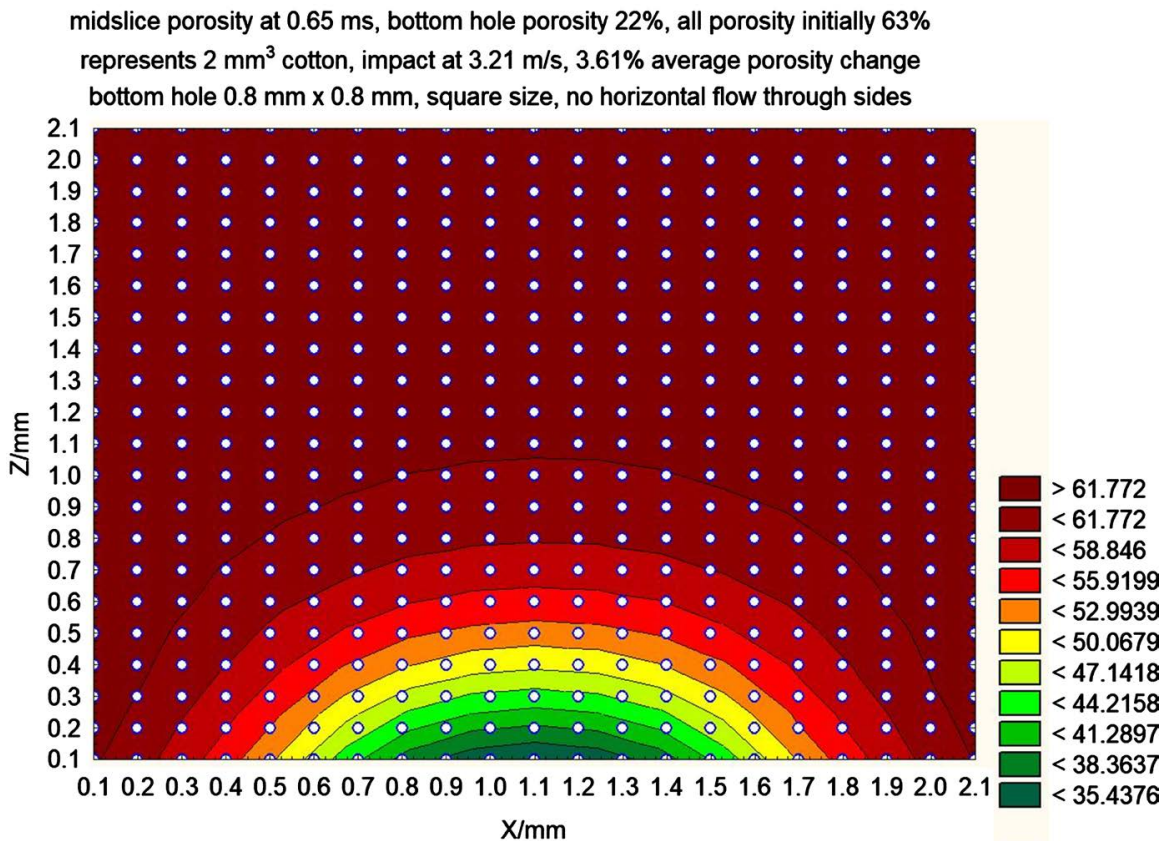

Figure 8. Impact drainage of wetted $2 \times 2 \times 2 \mathrm{~mm}^{3}$ cotton falling at $3.21 \mathrm{~m} / \mathrm{s}$ on a partially closed grating $\left(0.8 \times 0.8 \mathrm{~mm}^{2}\right.$ open $) 0.65 \mathrm{~ms}$ after impact, without horizontal flow through the side walls. 


\section{Conclusions}

The wash performance is more or less proportional to the perforated fraction of the drum area. A highly perforated drum may easily extend the open fraction to $1 / 2$ of the area and will lead to a significant increase of the speed of the wash process. Pumping suds from the annulus and drum will further increase the wash performance and reduce the drag on the drum. Water, chemicals, and energy reduction in the wash process help to better preserve and sustain the environment. Reduced wash time is beneficial for the end-user.

Technical realization of these proposed improvements is left to dedicated engineers.

\section{Acknowledgements}

I would like to thank Unilever for allowing this work to be published. I would like to thank Prof Dr Simeon D. Stoyanov (Unilever, WUR) and Mia Benjamin (Unilever) for their help.

\section{Conflicts of Interest}

The author declares no conflicts of interest regarding the publication of this paper.

\section{References}

[1] Sinner (1960) Über das Waschen in Haushaltswaschmaschinen. 2. Auflage Haus und Heim Verlag, Hamburg, 9-10.

[2] van den Brekel, L.D.M. (1987) Hydrodynamics and Mass Transfer in Domestic Drum-Type Fabric Washing Machines. Thesis, Delft.

[3] Lasic, E. (2014) Sustainable Use of Washing Machine: Modeling the Consumer Behavior Related Resources Consumption in Use of Washing Machines. Thesis Rheinischen Friedrich-Wilhelms-Universität, Bonn.

[4] Van der Weg (Peter), P.B. (2019) Poroelastic Modelling of Gravitational Compaction. Open Journal of Physical Chemistry, 9, 126-169. https://doi.org/10.4236/ojpc.2019.93008

[5] Van Wyk, C.M. (1946) Note on the Compressibility of Wool. Journal of the Textile Institute Transactions, 37, T285. https://doi.org/10.1080/19447024608659279

[6] Henderson, N., Brêttas, J.C. and Sacco, W.F. (2010) A Three-Parameter KozenyCarman Generalized Equation for Fractal Porous Media. Chemical Engineering Science, 65, 4432-4442. https://doi.org/10.1016/j.ces.2010.04.006

[7] Hossain, Z. and Cohen, A.J. (2012) Relationship among Porosity, Permeability, Electrical and Elastic Properties. SEG Las Vegas 2012 Annual Meeting. https://doi.org/10.1190/segam2012-1496.1

[8] Terzaghi, K. (1943) Theoretical Soil Mechanics, Wiley. https://doi.org/10.1002/9780470172766

[9] Kellett, J.R. (1974) Terzaghi's Theory of One Dimensional Consolidation of Soils and Its Application. Bureau of Mineral Resources, Geology and Geophysics, Record 1974/108. 


\section{Appendices}

\section{Appendix A. Theory: Article Movement in Horizontal Drum Washing}

A mathematical model for the drum-type washing machine is the basis for our calculations; see also Figure 1 for a side view on the drum. The laundry is considered a point mass $m$ describing a parabolic path. The drum is considered a horizontally mounted cylinder rotating on its axis with an angular speed $\omega$ $(\mathrm{rad} / \mathrm{s})$, frequency $f=\omega / 2 \pi$, and a linear speed of a point on its drum surface of $V_{c}=\omega R$, where $R$ the radius of the drum. A Cartesian coordinate system $(x, y)$ is chosen with $x$ horizontal, $y$ vertical, and the origin in the axis to describe the coordinates of the trajectory of the point mass. Gravity acts vertically downward opposite to the direction of the axis $y$. The acceleration of free fall is $g\left(9.81 \mathrm{~m} / \mathrm{s}^{2}\right)$. All chosen units conform to the MKSA system.

In the lower part of the drum, the point mass is pressed against the drum with a force $F=m \omega^{2} R-m g \sin \alpha$. Here $\alpha$ is the angle of the position of the point mass as rotated from the horizontal. At a certain height described with angle $\alpha=$ $\varphi$, the force becomes zero and the point mass must leave the drum to describe a parabolic path of free fall until it hits the lower part of the rotating drum at a point described with angle $\psi$.

At the leaving point $\left(x_{0}, y_{0}\right)$ the force is zero, hence $\sin \varphi=\frac{\omega^{2} R}{g}$. The value $\sin \varphi=\frac{\omega^{2} R}{g}$ is often denoted as the g-factor of the wash process. At the leaving point $\left(x_{0}, y_{0}\right)$ is $x_{0}=R \cos \varphi$ and $y_{0}=R \sin \varphi$, e.g. $v_{x}\left(x_{0}, y_{0}\right)=-\omega R \sin \varphi$ and $v_{y}\left(x_{0}, y_{0}\right)=\omega R \cos \varphi$. The parabola is described for any time $t$ since the mass point has detached by $x=R \cos \varphi-(\omega R \sin \varphi) t$ and

$y=R \sin \varphi+(\omega R \cos \varphi) t-\frac{1}{2} g t^{2}$. At the point of impact $\left(x_{e}, y_{e}\right)$ is $x=x_{e}=R \cos \psi$ and $y=y_{e}=R \sin \psi$ with $v_{x}\left(x_{e}, y_{e}\right)=v_{x}\left(x_{0}, y_{0}\right)=-\omega R \sin \varphi$, while $x_{e}=R \cos \varphi-(\omega R \sin \varphi) t_{e}$ and $y_{e}=R \sin \varphi+(\omega R \cos \varphi) t_{e}-\frac{1}{2} g t_{e}^{2}$ where $t_{e}$ the time of flight of the mass point along the section of the parabolic curve. Hence $t_{e}=\frac{\cos \varphi-\cos \psi}{\omega \sin \varphi}$. Substitution of this $t_{e}$ and $y_{e}=R \sin \psi$ in the $y_{e}$ equation gives: $R \sin \psi=R \sin \varphi+(\omega R \cos \varphi) \frac{\cos \varphi-\cos \psi}{\omega \sin \varphi}-\frac{1}{2} g\left(\frac{\cos \varphi-\cos \psi}{\omega \sin \varphi}\right)^{2}$, or using the g-factor: $\sin \varphi-\sin \psi+\cos \varphi\left(\frac{\cos \varphi-\cos \psi}{\sin \varphi}\right)-\frac{1}{2} \frac{1}{\sin \varphi}\left(\frac{\cos \varphi-\cos \psi}{\sin \varphi}\right)^{2}=0$. The physically relevant root of this equation is $\psi=2 \pi-3 \varphi$, hence $t_{e}=\frac{4 \cos \varphi \sin \varphi}{\omega}$. The total time $t_{t}$ for a closed path of the fabric consists of the time $t_{e}$ plus the time $t_{c}$ (corresponding with an angle of rotation of the drum of $4 \varphi$ and speed $v_{c}=\omega R$ ) that the article is following the drum: 
$t_{t}=\frac{4 \cos \varphi \sin \varphi}{\omega}+\frac{4 \varphi}{2 \pi} \frac{2 \pi R}{\omega R}=\frac{4}{\omega}(\cos \varphi \sin \varphi+\varphi)$. The angular frequency of an article is faster than that of the drum $\frac{\omega_{\text {article }}}{\omega}=\frac{\left(\frac{2 \pi}{t_{t}}\right)}{\omega}=\frac{2 \pi}{4(\cos \varphi \sin \varphi+\varphi)}$. The speed during the fall is $v_{x}=-\omega R \sin \varphi$ and $v_{y}=\omega R \cos \varphi-g t$ or a total speed during fall $v=\sqrt{(\omega R \sin \varphi)^{2}+(\omega R \cos \varphi-g t)^{2}}=\sqrt{\omega^{2} R^{2}-2 g t \omega R \cos \varphi+g^{2} t^{2}}$. The fall path has a total length of $s_{e}=\int_{0}^{t_{e}} v \mathrm{~d} t$.

Now suppose: $a=-\omega R \sin \varphi$ and $b=\omega R \cos \varphi$, thus $s_{e}=\int_{0}^{t_{e}} \sqrt{a^{2}+(b-g t)^{2}} \mathrm{~d} t=-\frac{1}{g} \int_{0}^{b-g t_{e}} \sqrt{a^{2}+u^{2}} \mathrm{~d} u$ or $s_{e}=-\frac{1}{g}\left[\frac{1}{2} u \sqrt{u^{2}+a^{2}}+\frac{1}{2} a^{2} \ln \left(u+\sqrt{u^{2}+a^{2}}\right)\right]_{u=b}^{u=\left(b-g t_{e}\right)}$. Suppose $c=b-g t_{e}$, then $s_{e}=-\frac{1}{g}\left[\frac{1}{2} c \sqrt{c^{2}+a^{2}}-\frac{1}{2} b \sqrt{b^{2}+a^{2}}+\frac{1}{2} a^{2} \ln \frac{\left(c+\sqrt{c^{2}+a^{2}}\right)}{\left(b+\sqrt{b^{2}+a^{2}}\right)}\right]$ where

$$
\begin{gathered}
c=b-g t_{e}=\omega R \cos \varphi-\frac{\omega^{2} R}{\sin \varphi} \frac{4 \cos \varphi \sin \varphi}{\omega}=-3 \omega R \cos \varphi, \sqrt{b^{2}+a^{2}}=\omega R, \\
\sqrt{c^{2}+a^{2}}=\omega R \sqrt{1+8 \cos ^{2} \varphi} \\
s_{e}=R \sin \varphi\left[\frac{3}{2}(\cos \varphi) \sqrt{1+8 \cos ^{2} \varphi}+\frac{1}{2} \cos \varphi\right.
\end{gathered}
$$

Hence

$$
\left.-\frac{1}{2}(\sin \varphi)^{2} \ln \frac{\left(-3 \cos \varphi+\sqrt{1+8 \cos ^{2} \varphi}\right)}{(\cos \varphi+1)}\right]
$$

The total path length is therefore $s_{t}=s_{e}+\frac{4 \varphi}{2 \pi} 2 \pi R=s_{e}+4 \varphi R$ or

$$
\begin{aligned}
s_{t}= & R(\sin \varphi)\left[\frac{4 \varphi}{\sin \varphi}+\frac{3}{2}(\cos \varphi) \sqrt{1+8 \cos ^{2} \varphi}+\frac{1}{2} \cos \varphi\right. \\
& \left.-\frac{1}{2}\left(\sin ^{2} \varphi\right) \ln \frac{\left(-3 \cos \varphi+\sqrt{1+8 \cos ^{2} \varphi}\right)}{(\cos \varphi+1)}\right]
\end{aligned}
$$

\section{Appendix B. Optimal Drum Design}

The formulas found in Appendix A are used to optimize the drum design to maximize the velocity change of fabric at impact with the drum.

We are interested in the speed just before and just after impact. First, we will focus on the speed just before the impact. The point mass is then at the end of the parabolic trajectory and has at that moment a speed given by $v_{x}\left(x_{e}, y_{e}\right)=-\omega R \sin \varphi$ 
and $v_{y}\left(x_{e}, y_{e}\right)=\left(\frac{\mathrm{d} y}{\mathrm{~d} t}\right)_{t=t_{e}}=\omega R \cos \varphi-g t_{e}=-3 \omega R \cos \varphi$. Just after impact, the mass point attains the speed of the drum $v_{x}\left(x_{e}, y_{e}\right)=-\omega R \sin \psi$ and $v_{y}\left(x_{e}, y_{e}\right)=\omega R \cos \psi$. The speed change in $x$-direction is $\Delta\left(v_{x}\right)=\omega R(-\sin \psi+\sin \varphi)$ and in $y$-direction $\Delta\left(v_{y}\right)=\omega R(\cos \psi+3 \cos \varphi)$.

The speed change on impact is therefore $\Delta v=\omega R \sqrt{(-\sin \psi+\sin \varphi)^{2}+(\cos \psi+3 \cos \varphi)^{2}}$

Introducing the explicit expression for the impact angle, we find for the speed change at impact $\Delta v=\omega R \sqrt{(\sin 3 \varphi+\sin \varphi)^{2}+(\cos 3 \varphi+3 \cos \varphi)^{2}}$. This may be simplified to $\Delta v=4 \omega R \cos ^{2} \varphi$. Since $\sin \varphi=\frac{\omega^{2} R}{g}$, we find $\Delta v=4 \omega R\left(1-\left(\frac{\omega^{2} R}{g}\right)^{2}\right)=4 \omega R-\frac{4 \omega^{5} R^{3}}{g^{2}}=\frac{4}{\omega}\left(\left(\omega^{2} R\right)-\frac{\left(\omega^{2} R\right)^{3}}{g^{2}}\right)$. By rearrangement: $\frac{\Delta v}{4 \omega R}=1-\left(\frac{\omega^{2} R}{g}\right)^{2}$. We may conclude that the g-factor $=\omega^{2} R / g$ and a term $\Delta v(\omega R)$ are the important dimensionless groups that determine the efficiency of a washing machine. The speed change as a function of drum radius and drum frequency for given acceleration of free fall of $g=9.81 \mathrm{~m} / \mathrm{s}^{2}$ is given in Figure B1.

For each frequency of the drum, there is a clear optimum in velocity change at a particular drum radius, and hence an optimal wash performance for horizontal axis drum-type washing machines at those particular conditions. We find the maximum velocity change at a given frequency by zeroing the first derivative of

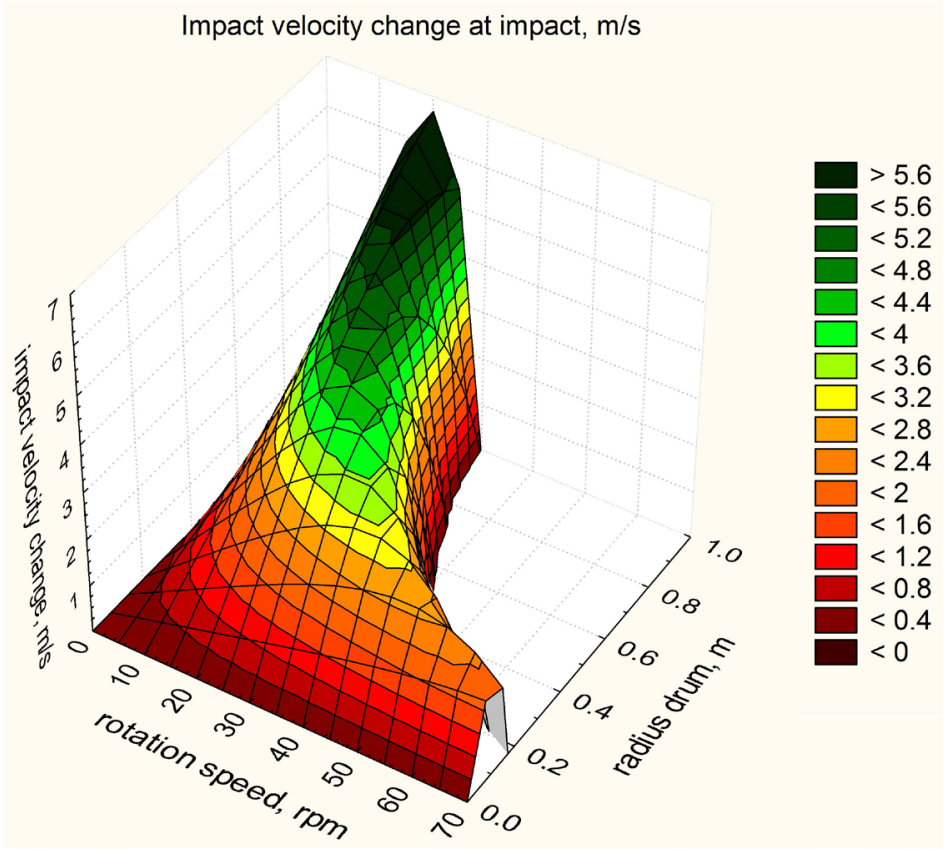

Figure B1. The impact velocity change $(\mathrm{m} / \mathrm{s})$ as function of speed of the drum (rpm) and the radius of the drum (m). 
$\Delta v$ to $R$ for a given and constant $\omega: R_{\max w r t \omega}=\frac{g}{\omega^{2} \sqrt{3}}$ and

$\Delta v_{\max w r t \omega}=\frac{8 g}{3 \omega \sqrt{3}} \approx \frac{1.54 \mathrm{~g}}{\omega}$. For $36.5 \mathrm{rpm}$, we find $R_{\max w r t \omega}=0.388 \mathrm{~m}$. For 30 $\operatorname{rpm}(0.5 \mathrm{~Hz})$, we find $R_{\max w r t \omega}=0.574 \mathrm{~m}$.

For each radius of the drum, there is a clear optimum in velocity change at a particular drum rotation speed, and hence an optimal wash performance for horizontal axis drum-type washing machines at those particular conditions.

The maximum velocity change at a given drum radius $R$ is found by zeroing the first derivative of $\Delta v$ to $\omega$ at a given and constant $R\left(\frac{\omega^{2} R}{g}\right)_{\text {optimum }}=\frac{1}{\sqrt{5}}$ and $\left(\frac{\Delta v}{\omega R}\right)_{\text {optimum }}=\frac{16}{5}$ or $\Delta v_{\text {optimum }}=\frac{16 g}{5 \omega \sqrt{5}} \approx \frac{1.431 \cdot g}{\omega}$

The maximum velocity change gives the best wash performance, e.g. as represented by points along the top of the ridge in Figure B1. For a given radius $R$ the optimum design with maximum wash performance is

$$
\left(g_{\text {factor }}\right)_{\text {optimum }}=(\sin \varphi)_{\text {optimum }}=\left(\frac{\omega^{2} R}{g}\right)_{\text {optimum }}=\frac{1}{\sqrt{5}} \approx 0.45
$$

The optimum angle of fabric detachment from the drum surface during rotation occurs at an angle of $\varphi=\arcsin (1 / \sqrt{5})=26.7$ degree with respect to the horizontal middle plane of the drum. The optimum angle of impact is therefore $360-3 \varphi=280$ degrees: the optimum impact on the drum occurs at 10 degrees rotation beyond the lowest point, where the drum's surface point is moving to the right in a slightly upward direction.

The fall trajectory length was

$$
s_{e}=R \sin \varphi\left[\frac{3}{2}(\cos \varphi) \sqrt{1+8 \cos ^{2} \varphi}+\frac{1}{2} \cos \varphi\right.
$$

$$
\left.-\frac{1}{2}(\sin \varphi)^{2} \ln \frac{\left(-3 \cos \varphi+\sqrt{1+8 \cos ^{2} \varphi}\right)}{(\cos \varphi+1)}\right]
$$

and corresponds for the optimum angle to a fall path length

$$
\begin{aligned}
S_{e, \text { optimum }} & =R \frac{1}{\sqrt{5}}\left[\frac{3}{2} \frac{2}{\sqrt{5}} \sqrt{1+8 \frac{4}{5}}+\frac{1}{2} \frac{2}{\sqrt{5}}-\frac{1}{2} \frac{1}{5} \ln \frac{\left(-3 \frac{2}{\sqrt{5}}+\sqrt{1+8 \frac{4}{5}}\right)}{\left(\frac{2}{\sqrt{5}}+1\right)}\right] \text { while the } \\
& =2.0082 \cdot R
\end{aligned}
$$

optimum total trajectory length for the optimum $\varphi=\arcsin (1 / \sqrt{5})$ is

$$
\begin{aligned}
s_{t} & =\left(s_{e}+4 \varphi R\right)_{\text {optimum }} \\
& =\frac{R}{5 \sqrt{5}}\left[3 \sqrt{37}+\sqrt{5}-\frac{1}{2} \cdot \ln \frac{(-6+\sqrt{37})}{(2+\sqrt{5})}\right]+4 \varphi R . \text { The optimum angular frequen- } \\
& \approx 3.8628 \cdot R
\end{aligned}
$$


cy and frequency of an article in the drum is significantly larger than that of the drum itself:

$$
\frac{\omega_{\text {article }}}{\omega_{\text {drum }}}=\frac{f_{\text {article }}}{f_{\text {drum }}}=\left(\frac{2 \pi}{4(\cos \varphi \sin \varphi+\varphi)}\right)=\frac{\pi}{\frac{4}{5}+2 \arcsin \left(\frac{1}{\sqrt{5}}\right)} \approx 1.812
$$

The optimum trajectory is shown in Figure B2.

Introducing the optimum condition in the speed equation leads to an optimal velocity change on impact of $\Delta v_{\text {optimum }}=\frac{16 g}{5 \omega \sqrt{5}} \approx \frac{14.04}{(\omega / \mathrm{rad} / \mathrm{s})} \mathrm{m} / \mathrm{s}=\frac{134.1}{(f / \mathrm{rpm})} \mathrm{m} / \mathrm{s}$. We may compare these data in Table B1 with the data drum diameter/frequency for the main wash in commercial washing machines for in-home use.

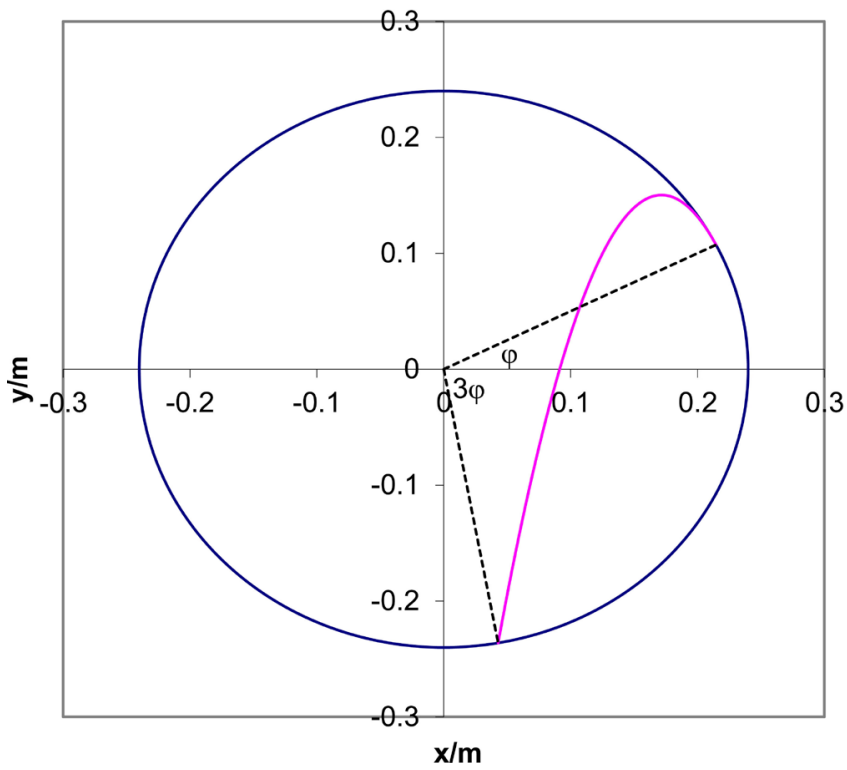

Figure B2. The optimum trajectory of laundry in a rotating drum washing machine of 48 $\mathrm{cm}$ internal diameter that should, for the optimum conditions at that radius, be operated at a rotation speed of $40.8 \mathrm{rpm}$.

Table B1. Design parameters of commercial washing machines compared with optimum conditions for maximum impact velocity change.

\begin{tabular}{ccccc}
\hline Type & Type & $\begin{array}{c}\text { diameter } \\
\mathrm{cm}\end{array}$ & $\begin{array}{c}\text { Operation speed } \\
\mathrm{rpm}\end{array}$ & $\begin{array}{c}\text { Optimum speed } \\
\mathrm{rpm}\end{array}$ \\
\hline Constructa & & 50 & 35 & 40 \\
Miele & W698 & 48 & $55(5 \mathrm{rev}) \& 40(4 \mathrm{rev})$ & 40.8 \\
Miele & Novotronic W933 & 48 & 54 \& 39 & 40.8 \\
& & 48 & $29($ wool $)$ & 40.8 \\
AEG & Turna & 48 & 40 & 40.8 \\
AEG & Bella & 48 & 55 & 40.8 \\
AEG & Lavamat & 48 & 55 & 40.8 \\
Zanussi & IZ12 & 46 & 53.4 & 41.7 \\
\hline
\end{tabular}


We observe that for the washing machines of which we have sufficient data, several are not operating near the optimal frequency for good wash efficiency for a small load.

We have found very simple formulas that help to optimize the design of horizontal axis washing machines to maximize the ultimate wash performance potential. The maximum velocity change corresponds to each cloth with the maximum impulse $(\Delta p=m \Delta v)$ change. Since this impulse change is exerted in a very short time, this also corresponds with the maximum force $F=\Delta p / \Delta t$ on the wet fabric, thus with the maximum relative speed of the wash liquor with respect to the fabric, and therefore with maximum potential wash performance.

Heavily loaded machines will create a bed of fabric, reducing the effective radius of rotation for the fabric at the top of the bed. The articles will then rotate and tumble backward, creating an effective lower rotation speed of the centre of mass of the articles compared to that of the drum, resulting in lower wash performance, and maybe another optimum rpm.

\section{Appendix C. Multidimensional Simulation of Creeping Flow}

Steady state 3-D porosity distribution for boundary conditions representing a cube of cotton with imposed porosity profile on boundaries.

Differential equation for porosity $\phi$ :

$\partial \phi / \partial t=$ coef $*\left(\partial^{2} \phi / \partial x^{2}+\partial^{2} \phi / \partial y^{2}+\partial^{2} \phi / \partial z^{2}\right), \quad D_{s}=$ coef $=k_{0} \varepsilon_{0} / \mu=$ permeability ${ }^{\star}$ elasticity/viscosity. The permeability, elasticity, and viscosity are uniform and constant during flow. Porosity change and flow in a cube of elastic wetted fabric, pressurized and draining through holes in the base under lower pressure. Box is a $[i, j, k]=(x, y, z)=[N x \times N y \times N z]=x \mathrm{dim}^{3} \mathrm{~mm}^{3}$ grid spacing along each side. A Gauss-Seidel iteration is employed until steady state, e.g. either the sum of absolute values of the porosity $\phi(i, j, k)$ adaptations from their last computed values falls below a small quantity epsmax, or, the number of iterations exceeds itmax (Ref. [C1]). An example of FORTRAN coding:

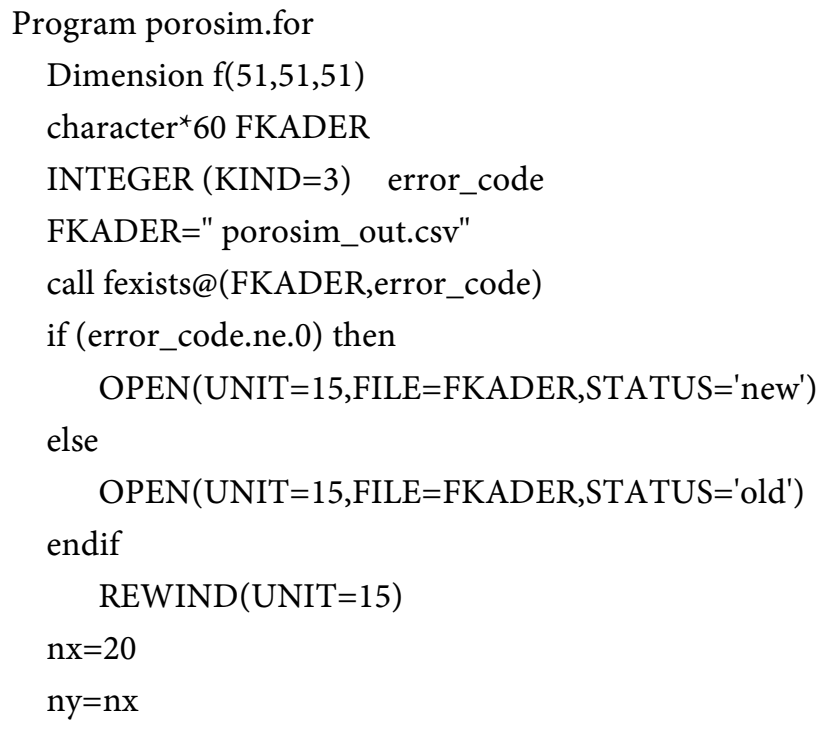




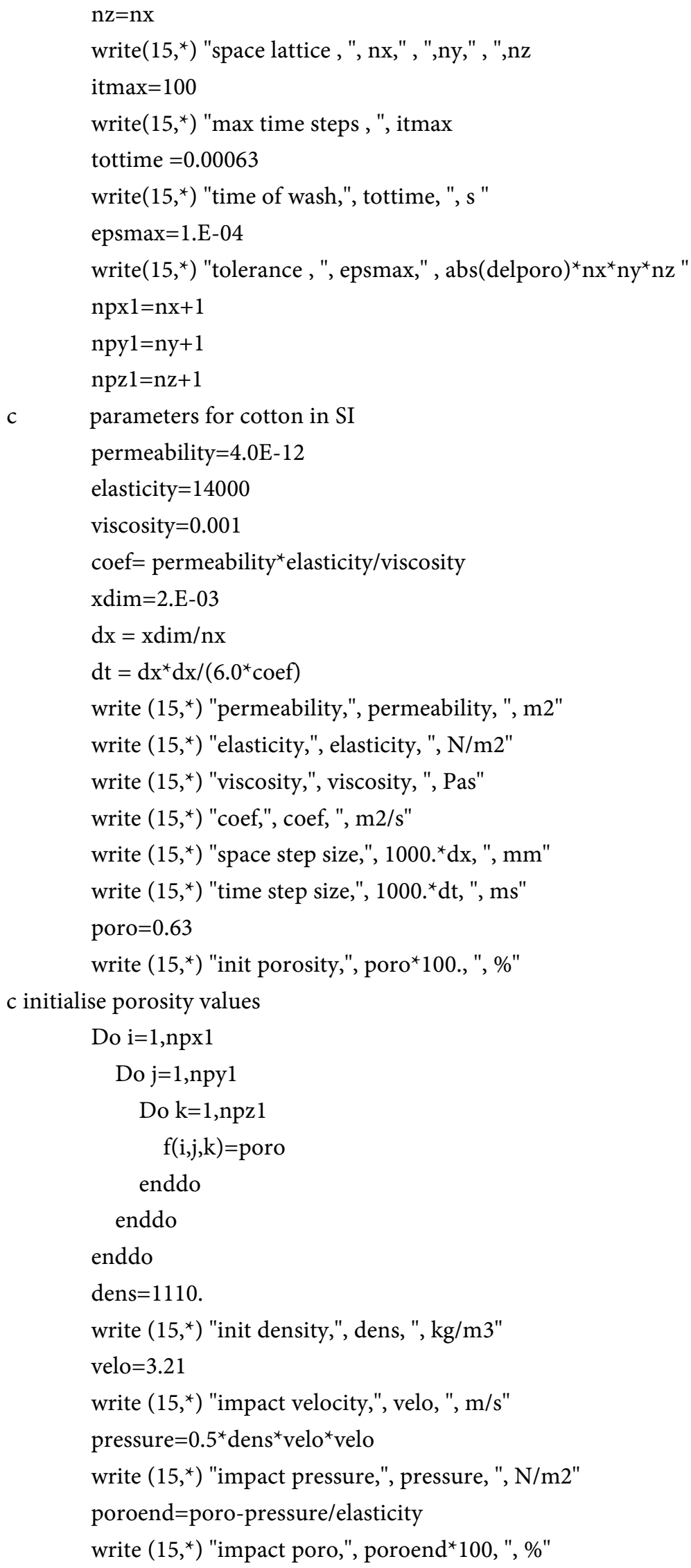


c initialise holes in the input layer

$c$ half thickness of hole

$$
\begin{aligned}
& \text { nrx=4 } \\
& \text { nxmid=(npx1+1)/2 } \\
& \text { nxstart=nxmid-nrx } \\
& \text { nxend=nxmid+nrx } \\
& \text { If (nxstart.lt.1) nxstart=1 } \\
& \text { If (nxend.gt.npx1)nxend=npx1 } \\
& \text { write }\left(15,{ }^{*}\right) \text { "nxstart,", nxstart } \\
& \text { write }\left(15,{ }^{*}\right) \text { "nxend,", nxend } \\
& \text { Do } \mathrm{i}=\mathrm{nxstart}, \mathrm{nxend} \\
& \quad \text { Do } \mathrm{j}=\mathrm{nxstart}, \mathrm{nxend} \\
& \quad \mathrm{k}=1 \\
& \quad \mathrm{f}(\mathrm{i}, \mathrm{j}, \mathrm{k})=\text { poroend } \\
& \quad \text { enddo } \\
& \text { enddo }
\end{aligned}
$$

c calculate successively better approximations

$c$ for porosity at all grid points, iterating

$c$ until satisfactory convergence is achieved

$$
\text { iter }=0
$$

3 iter $=$ iter +1

$$
\begin{aligned}
& \text { eps }=0 \\
& \text { Do } i=2, n x \\
& \text { Do } j=2, n y
\end{aligned}
$$$$
\text { Do } \mathrm{k}=2, \mathrm{nz}
$$$$
\text { Holdf }=f(i, j, k)
$$$$
f(i, j, k)=(f(i-1, j, k)+f(i+1, j, k)+f(i, j-1, k)
$$$$
+\quad+\mathrm{f}(\mathrm{i}, \mathrm{j}+1, \mathrm{k})+\mathrm{f}(\mathrm{i}, \mathrm{j}, \mathrm{k}-1)+\mathrm{f}(\mathrm{i}, \mathrm{j}, \mathrm{k}+1)) / 6
$$$$
\text { eps }=\text { eps }+A B S(f(i, j, k)-\text { Holdf })
$$

enddo

enddo

enddo

c do not allow horizontal flow through side planes

$$
\begin{aligned}
& \text { Do } i=1, n p x 1 \\
& \text { do } k=2, n p z 1 \\
& \quad f(i, 1, k)=f(i, 3, k) \\
& \quad f(i, n p y 1, k)=f(i, n p y 1-2, k) \\
& \text { enddo } \\
& \text { enddo }
\end{aligned}
$$

Do $\mathrm{j}=1$, npy 1

do $\mathrm{k}=2, \mathrm{npz} 1$ 


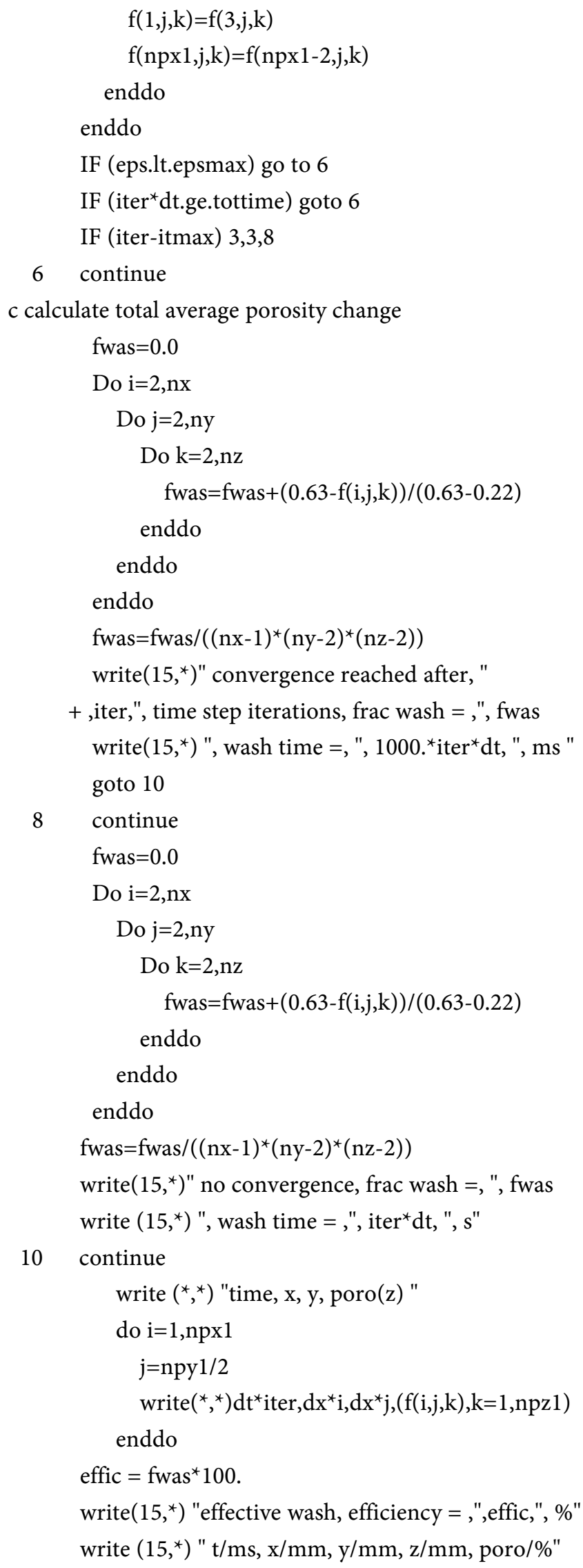




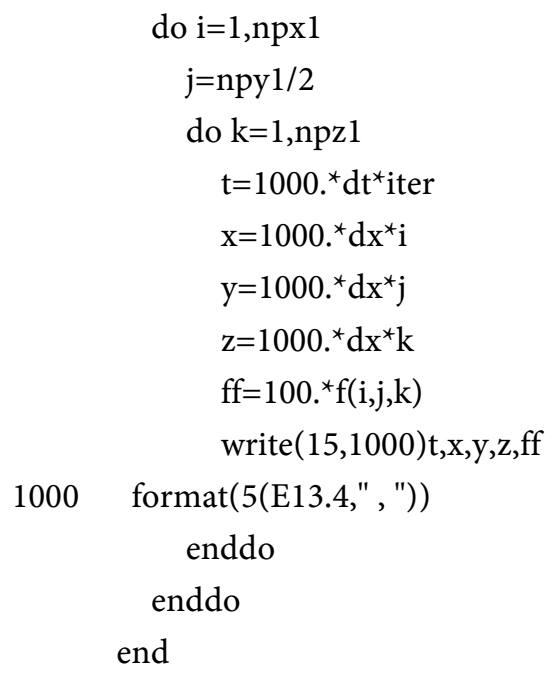

\section{Reference Appendix C}

[C1] Carnahan, B., Luther, H.A. and Wilkes, J.O. (1969) Applied Numerical Methods. Example 7.6, 486-490, J. Wiley \& Sons, New York. 